\title{
The Incorporation of Elaidate, Oleate and Straight-chain Saturated Fatty Acids by Mycoplasma Strain Y
}

\author{
By A. W. RODWELL \\ CSIRO Division of Animal Health, Animal Health Research Laboratory, \\ Private Mail Bag no. I, P.O. Parkville, Melbourne, Victoria 3052, Australia
}

\author{
(Accepted for publication 5 June 197I)
}

\begin{abstract}
SUMMARY
Mycoplasma strain Y grew well in a partly defined medium containing charcoaltreated bovine serum albumin (BSA) with added elaidate, elaidate plus oleate, or elaidate plus a saturated fatty acid containing Io to I5, 22 or 24 carbon atoms. With elaidate plus a saturated acid containing I 6 to 20 carbon atoms there was good growth after a variable lag period. When elaidate was the only fatty acid supplied it comprised from 93 to $95 \%$ of the total fatty acids in stationary-phase mycoplasmas, the remainder being derived from contaminating fatty acids in the medium. There was no isomerization of elaidate to oleate. When grown with elaidate plus a shorter-chain saturated acid $\left(\mathrm{C}_{10}, \mathrm{C}_{12}\right)$ or with a long-chain acid $\left(\mathrm{C}_{22}, \mathrm{C}_{24}\right)$, elaidate was incorporated preferentially during the early stages of growth, increasing proportions of the saturated acids being incorporated during the later stages. When grown with elaidate plus a saturated acid of intermediate chain length $\left(\mathrm{C}_{14}\right.$ to $\left.\mathrm{C}_{16}\right)$, or with elaidate plus oleate, both fatty acids were incorporated in approximately equal proportions throughout growth. The preferential incorporation of elaidate in the early growth stages with some pairs of fatty acids is explained by a competition between the two fatty acids for the BSA binding sites.
\end{abstract}

\section{INTRODUCTION}

Mycoplasma strain Y grew well in a partly defined medium (medium C) with the addition of pairs of fatty acids: one a monoenoic acid, the other a saturated acid. The chain length, the position and the configuration of the double bond in the monoenoic acid supplied, each influenced the range of saturated fatty acids compatible with growth (Rodwell, 1967). Elaidate could apparently fulfil the functions of both a saturated and an unsaturated acid, because with it there was good growth in the absence of added saturated fatty acid. Octadecenoate then comprised about $97 \%$ of the fatty acids in the lipids; when this was supplied together with a saturated acid, both were incorporated (Rodwell, 1968). Further work on the nutrition of sterol-requiring mycoplasmas (Rodwell, 1969) indicated that the dispersion of cholesterol in an assimilable form in medium $\mathrm{C}$ depended on an interaction with fatty acids. Certain samples of bovine serum albumin, which is present in medium $\mathrm{C}$ to bind fatty acids, may bind them so firmly that their interaction with cholesterol is prevented, and the mycoplasmas may then undergo lysis during growth caused by a deficiency of cholesterol. To overcome this difficulty, a pronase-treated, defatted serum protein fraction with cholesterol-dispersing activity was included in medium $\mathrm{C} \mathrm{2}$. In this paper, the results of fattyacid analyses of the lipids of strain $\mathrm{Y}$ harvested at different stages of growth in medium $\mathrm{C}_{2}$ are reported. With some pairs (elaidate plus a saturated acid of chain length $\mathrm{C}_{14}$ to $\mathrm{C}_{16}$, or oleate) both acids were incorporated in approximately equal proportions throughout growth. 
With other pairs elaidate was incorporated preferentially in the early stages of growth, and the saturated acid during the later stages. This is attributed to competition between the two fatty acids for the BSA binding sites.

\section{METHODS}

Organisms and growth conditions. Mycoplasma strain $\mathrm{Y}$ has been described in previous publications from this laboratory. Strain $80 \mathrm{I}$ is serologically related to Mycoplasma mycoides (Cottew, I970) and was isolated in this laboratory from the eye of a cow.

Medium C 2 was as described previously (Rodwell, I969). Fatty acids were added as solutions of their sodium salts to a total concentration of $0.1 \mathrm{mM}$, and charcoal-treated BSA to a concentration of $I \cdot 6$ g./1., i.e. the initial molar ratio fatty acid:BSA was 3:7.

The inoculum was prepared from cultures grown in BVFOS medium (Turner, Campbell $\&$ Dick, 1935). The cultures were centrifuged and the mycoplasmas suspended in an equal volume of $0.02 \mathrm{M}$-tris- $\mathrm{HCl}$ buffer $\left(\mathrm{pH} 7.0\right.$ ) containing $0.01 \mathrm{M}-\mathrm{MgSO}_{4}(\mathrm{TM})$. The suspensions were diluted $\mathrm{I} / \mathrm{I} 00$ in TM and one drop of the diluted suspensions $\left(5 \times 10^{5}\right.$ colony-forming units) was inoculated into $5 \mathrm{ml}$. volumes of medium $\mathrm{C} 2$ in optically matched tubes. Growth was measured at intervals during incubation at $37^{\circ}$ by extinction at $660 \mathrm{~nm}$.

Analysis of fatty acids. Replicate cultures were combined for analysis (total volume, usually $\mathrm{I} 0 \mathrm{ml}$.). The organisms were harvested by centrifugation at $\mathrm{I} 6,000 \mathrm{~g}$, washed once

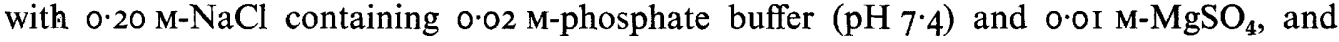
resuspended in a small volume of the same solution. The suspension was treated with I 9 vol. chloroform + methanol $(2+\mathrm{I}, \mathrm{V} / \mathrm{v})$ for $\mathrm{I} h$. at room temperature, filtered through glass wool, washed with $0.2 \mathrm{vol}$. o. I $\mathrm{M}-\mathrm{KCl}$, and the lower phase-dried with $\mathrm{Na}_{2} \mathrm{SO}_{4}$.

Fatty acid methyl esters were prepared by alkaline methanolysis. The lipids were dissolved in chloroform and $0.5 \mathrm{vol}$. of $0.2 \mathrm{M}-\mathrm{Na}$ methoxide added. After $\mathrm{I} \mathrm{h}$. at room temperature, 0.2 vol. of $0.4 \mathrm{M}$-aqueous acetic acid was added. The lower phase was washed with water and dried over $\mathrm{Na}_{2} \mathrm{SO}_{4}$. Fatty-acid methyl esters were separated from cholesterol by chromatography on Florisil columns (Kopaczyk \& Radin, I965). The lipids after methanolysis were applied to the column in hexane + benzene $(9+\mathrm{I}, \mathrm{v} / \mathrm{v})$ and fatty acid methyl esters eluted with the same solvent.

Fatty acids were determined by gas-liquid chromatography (g.l.c.). Methyl pentadecanoate or methyl arachidate were used as internal standards and were in most cases added to the lipid extract before methanolysis.

To determine oleate in the presence of elaidate, part of the methyl ester fraction was chromatographed on thin layers of silica gel G, impregnated with silver nitrate (Ag-TLC) and developed in diethylether + light petroleum $(5+95, \mathrm{v} / \mathrm{v})$ as described by Morris (1964). Methyl esters were located by spraying with a solution of $2^{\prime}, 7^{\prime}$-dichlorofluorescein in methanol and viewing under an ultraviolet lamp. The bands containing the esters of saturated acids, trans-monoenes and cis-monoenes were scraped from the plates, the esters eluted with chloroform and determined by g.l.c. after the addition of an appropriate amount of standard. Part of the methyl ester fraction was analysed directly and the results used to correct for losses on TLC.

\section{RESULTS}

\section{Growth with elaidate or oleate and saturated fatty acids}

Strain Y grew poorly, and then only after a prolonged lag period, in medium $\mathrm{C}_{2}$ in the absence of added fatty acids. It grew well on the addition of elaidate alone, or of elaidate and equimolar concentrations of caprate, laurate, myristate or pentadecanoate. With palmitate, 
heptadecanoate or stearate normal growth occurred with an increasing lag period; with arachidate the lag period was less, and with still longer chain acids (behenate, lignocerate) the growth curves were similar to those with elaidate alone or elaidate plus a shorter chain acid (Fig. I). (For clarity, growth curves with selected pairs only are included in Fig. I.)

When oleate was the only fatty acid added, there was early growth followed by rapid lysis. With oleate plus a saturated acid containing I 2 to 18 carbon atoms, or with oleate plus elaidate, there was good growth. The fastest growth rate was obtained with oleate plus heptadecanoate. With oleate and caprate, arachidate or saturated acids of longer chain length the growth curves were similar to that with oleate alone (Fig. 2).

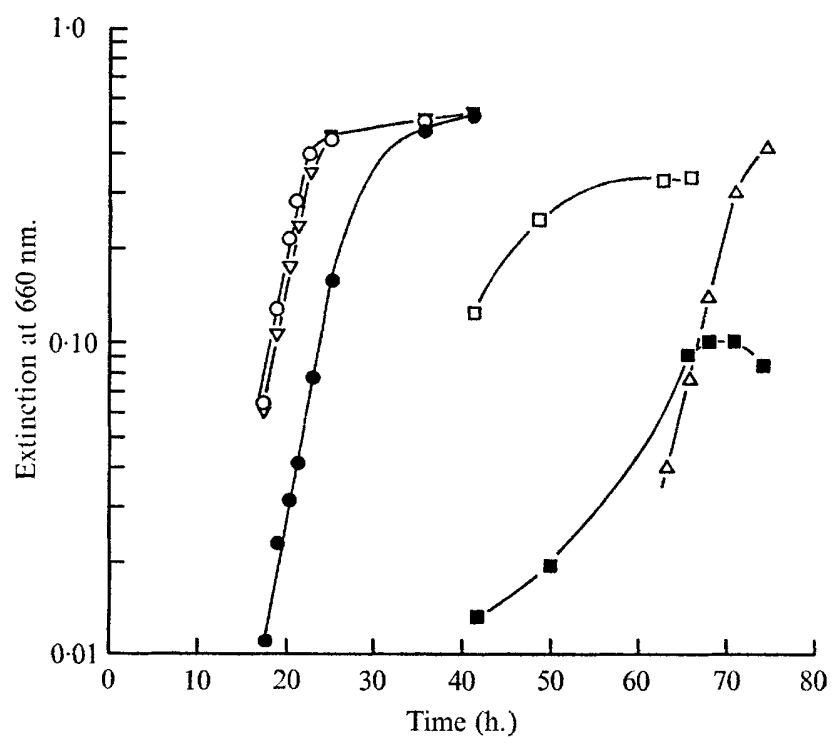

Fig. I

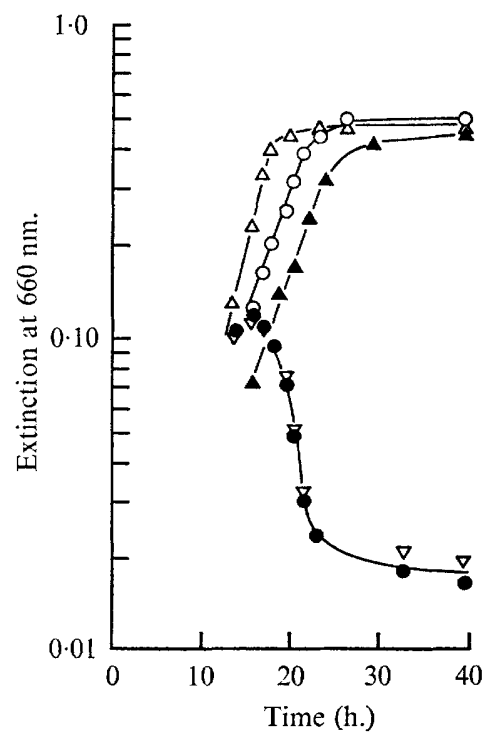

Fig. 2

Fig. I. Growth of Mycoplasma strain Y in medium C2 with elaidate, or elaidate plus saturated fatty acids. $\square$, No added fatty acids; $\bigcirc$, elaidate; $\nabla$, elaidate plus behenate; $\odot$, elaidate plus palmitate; $\square$, elaidate plus arachidate; $\triangle$, elaidate plus stearate.

Fig. 2. Growth of Mycoplasma strain $\mathrm{y}$ in medium $\mathrm{C}_{2}$ with oleate plus saturated fatty acids or elaidate. $\bullet$, Oleate; $\triangle$, oleate plus palmitate; 0 , oleate plus laurate; $\boldsymbol{\Delta}$, oleate plus elaidate; $\nabla$, oleate plus arachidate.

\section{Fatty-acid incorporation}

Cultures were analysed during the exponential growth phase (extinction $=0.15$ to 0.25 ), after exponential growth had ceased and in the stationary phase.

Strains $80 \mathrm{I}$ and $\mathrm{Y}$ were grown in medium in which elaidate was the only fatty acid added, and the fatty-acid composition of the lipids determined after separating the methyl esters of cis and trans monoenes by Ag-TLC. The TLC plates after spraying with dichlorofluorescein showed intense fluorescent bands migrating like methyl elaidate, and easily visible bands migrating like methyl palmitate. There was no detectable fluorescence in the cis monoene regions, but these were scraped from the plates and analysed. Elaidate accounted for 93 to $95 \%$ of the total fatty acids in the lipids, palmitate and stearate accounting for most of the remainder. Only traces $(0.3$ to $0.5 \%)$ to octadecenoic acid were found in the eluates from the cis monoene areas of the plates (Table I). 
Strain $\mathrm{Y}$ was examined after growth with equimolar concentrations of elaidate plus saturated acids. With short-chain acids $\left(\mathrm{C}_{10}, \mathrm{C}_{12}\right)$ elaidate was incorporated exclusively during exponential growth. Caprate and laurate were incorporated during the later stages and accounted for $\mathrm{I} 2$ and $22 \%$ of the total esterified acids in the lipids of stationary phase mycoplasmas (Table 2). With elaidate and a saturated acid of intermediate chain length $\left(\mathrm{C}_{14}, \mathrm{C}_{15}, \mathrm{C}_{16}\right)$, both acids were incorporated at about equal rates throughout growth. The

Table I. Fatty-acid composition of the lipids of Mycoplasma strains $\mathrm{Y}$ and $80 \mathrm{O}$ after growth in medium $\mathrm{C} 2$ with elaidate

\begin{tabular}{|c|c|c|c|c|c|c|}
\hline \multirow[b]{2}{*}{ Strain } & \multirow{2}{*}{$\begin{array}{l}\text { Incubation } \\
\text { (h.) }\end{array}$} & \multirow{2}{*}{$\begin{array}{l}\text { Culture } \\
\text { extinction }\end{array}$} & \multicolumn{4}{|c|}{ Fatty-acid composition (mole \%) } \\
\hline & & & $16: 0$ & $18: 0$ & 18: I cis & I8: I trans \\
\hline 801 & $\begin{array}{l}22 \cdot 2 \\
37 \cdot 7\end{array}$ & $\begin{array}{l}0.17 \\
0.46\end{array}$ & $\begin{array}{l}3 \\
2\end{array}$ & $\begin{array}{l}3 \\
4\end{array}$ & $\begin{array}{l}0.5 \\
0.3\end{array}$ & $\begin{array}{l}94 \\
94\end{array}$ \\
\hline $\mathbf{Y}$ & $\begin{array}{l}17 \cdot 2 \\
23 \cdot 5 \\
39 \cdot 3\end{array}$ & $\begin{array}{l}0.2 I \\
0.39 \\
0.46\end{array}$ & $\begin{array}{l}3 \\
2 \\
2\end{array}$ & $\begin{array}{l}4 \\
3 \\
4\end{array}$ & $\begin{array}{l}0.4 \\
0.2 \\
0.3\end{array}$ & $\begin{array}{l}93 \\
95 \\
94\end{array}$ \\
\hline
\end{tabular}

Table 2. Incorporation of elaidate plus laurate, pentadecanoate or behenate during growth of Mycoplasma strain $\mathbf{Y}$

\begin{tabular}{lccccc} 
& \multicolumn{4}{c}{ Fatty acids in lipids (mole \%) } \\
Fatty acids supplied & $\begin{array}{c}\text { Incubation } \\
\text { (h.) }\end{array}$ & $\begin{array}{c}\text { Culture } \\
\text { extinction }\end{array}$ & $\begin{array}{c}\text { Added } \\
\text { saturated }\end{array}$ & I 8: I & Others* \\
Elaidate+ laurate & $19 \cdot 0$ & 0.27 & $<1$ & 95 & 5 \\
& $24 \cdot 3$ & 0.46 & 9 & 87 & 4 \\
Elaidate+ pentadecanoate & $48 \cdot 0$ & 0.51 & 22 & 74 & 4 \\
& $27 \cdot 8$ & 0.25 & 44 & 52 & 5 \\
Elaidate+ behenate & 40.5 & 0.43 & 44 & 51 & 5 \\
& $48 \cdot 3$ & 0.42 & 44 & 52 & 4 \\
& $18 \cdot 3$ & 0.21 & 5 & 90 & 5 \\
& $23 \cdot 3$ & 0.44 & 22 & 73 & 5 \\
& $39 \cdot 4$ & 0.48 & 49 & 48 & 3
\end{tabular}

* Mainly $16: 0$ and $18: 0$, traces of $14: 0,16: 1$ and $18: 2$.

results with elaidate and pentadecanoate are shown in Table 2 . With elaidate and longchain acids $\left(\mathrm{C}_{22}, \mathrm{C}_{24}\right)$, elaidate was incorporated preferentially during exponential growth, and the saturated acid during the later stages. Behenate and lignocerate accounted for approximately 50 and $30 \%$, respectively, of the total in the lipids of stationary phase mycoplasmas. The results with elaidate and behenate are shown in Table 2.

When strain Y was grown with elaidate plus oleate, Ag-TLC plates of the methyl esters showed fluorescent bands of about equal intensity in both cis and trans monoene positions, and a faintly fluorescent band in the saturated acid position. The proportions of the cis and trans isomers in the lipids did not change markedly during growth, although a higher proportion of elaidate was incorporated, particularly in the early stages (Table 3).

To determine whether the abrupt change in the fatty acid incorporated with certain pairs (e.g. elaidate plus behenate, Table 2) was due to exhaustion of available elaidate, strain $\mathbf{Y}$ was grown with elaidate + behenate in initial molar ratios of $I: 3, I: I$ and $3: I$, the total fatty-acid concentration and the initial ratio of fatty acid to BSA being kept constant. Growth in the cultures with an initial ratio of elaidate to behenate of I:I and 3:I was 
similar, but was slower in the cultures with the higher initial proportion of behenate. Mycoplasmas were analysed at the times shown (Table 4), and the amounts of each fatty acid remaining at each time interval determined by difference. With an initial elaidate to behenate ratio of $I: 3$, behenate was incorporated earlier and to a greater extent, and in the later stages it was incorporated exclusively, despite the fact that some elaidate remained unincorporated.

Table 3. Incorporation of elaidate and oleate during growth of Mycoplasma strain $\mathbf{Y}$

\begin{tabular}{|c|c|c|c|c|c|}
\hline \multirow{2}{*}{$\begin{array}{l}\text { Incubation } \\
\text { (h.) }\end{array}$} & \multirow{2}{*}{$\begin{array}{l}\text { Culture } \\
\text { extinction }\end{array}$} & \multicolumn{3}{|c|}{ Fatty-acid composition (mole \%) } & \multirow{2}{*}{$\begin{array}{c}\text { Total incorporated } \\
(\mu \mathrm{mole} / 100 \mathrm{ml} . / \text { culture })\end{array}$} \\
\hline & & 18: I cis & 18: I trans & Others & \\
\hline $20 \cdot 5$ & 0.19 & 38 & 58 & 4 & 2.5 \\
\hline 25.0 & 0.35 & $4 \mathrm{I}$ & 52 & 7 & $4 \cdot 3$ \\
\hline $39 \cdot 8$ & 0.42 & 43 & 49 & 8 & $4 \cdot 6$ \\
\hline
\end{tabular}

Table 4. The effect of various proportions of elaidate and behenate in medium $C 2$ on the amounts incorporated during growth of Mycoplasma strain $\mathrm{Y}$

\begin{tabular}{|c|c|c|c|c|}
\hline \multirow[b]{2}{*}{$\begin{array}{l}\text { Incubation } \\
\text { (h.) }\end{array}$} & \multirow[b]{2}{*}{$\begin{array}{l}\text { Culture } \\
\text { extinction }\end{array}$} & \multicolumn{2}{|c|}{$\begin{array}{l}\text { Fatty acids in medium } \\
\qquad(\mu \mathrm{mole} / \mathrm{I} 00 \mathrm{ml} .)\end{array}$} & \multirow{2}{*}{$\begin{array}{l}22: 0 \text { in } \\
\text { lipids (mole } \\
\text { proportion }\end{array}$} \\
\hline & & I 8: It & $22: 0$ & \\
\hline 0 & - & 2.5 & 7.5 & - \\
\hline 40 & 0.15 & $\mathrm{I} \cdot 4$ & 7.0 & $0.3 \mathrm{I}$ \\
\hline 50 & 0.28 & $1 \cdot 0$ & 5.6 & 0.56 \\
\hline 63 & 0.35 & I. 0 & $4 \cdot 7$ & 0.65 \\
\hline 0 & - & 5.0 & 5.0 & - \\
\hline 28 & 0.26 & $3 \cdot 0$ & 4.8 & 0.10 \\
\hline 40 & 0.44 & $2 \cdot I$ & $2 \cdot 8$ & 0.43 \\
\hline 50 & 0.44 & 2.0 & $\mathrm{I} \cdot 8$ & 0.50 \\
\hline 0 & - & 7.5 & 2.5 & - \\
\hline 28 & 0.29 & $4 \cdot 3$ & $2 \cdot 3$ & 0.06 \\
\hline 40 & 0.44 & $3 \cdot 4$ & $I \cdot 5$ & 0.20 \\
\hline 50 & 0.43 & $2 \cdot 9$ & $I \cdot 2$ & 0.22 \\
\hline
\end{tabular}

\section{DISCUSSION}

The delayed growth in medium $\mathrm{C}_{2}$ in the absence of added fatty acids, and the presence of fatty acids (totalling 4 to $8 \%$ ) other than those added in the lipids of mycoplasmas grown with suitable fatty-acid supplement were due to the presence of contaminating fatty acids in medium $\mathrm{C}$ 2. The results support the conclusion that mycoplasma strain $\mathrm{Y}$ is incapable of isomerizing monoenoic acids, desaturating saturated acids, or of altering the chain length of either. Further evidence in support of this conclusion was obtained by the failure to obtain any incorporation of carbon from $\left[{ }^{14} \mathrm{C}\right]$ glucose (as a potential source of acetyl groups for fatty-acid synthesis) into fatty acids in mycoplasmas grown in fatty-aciddeficient medium (A. W. Rodwell, unpublished).

The abrupt change in fatty-acid composition during growth with certain pairs of fatty acids (e.g. elaidate plus behenate or laurate) is attributed to the different binding affinities of the two fatty acids for the BSA binding sites. Elaidate is preferred, and when freely available is incorporated almost exclusively, but in the later stages of growth when the ratio of BSA: fatty acids has declined, the remaining elaidate is bound at the stronger binding sites and is less readily available. Spector, Steinberg \& Tanaka (1965) showed that the initial rate of palmitate uptake by Ehrlich ascites tumour cells was determined by the molar ratio 
of fatty acid to BSA, becoming maximal at ratios greater than 3: I. At lower fatty-acid:BSA ratios the rate of uptake was proportional to the calculated concentration of unbound fatty acid in equilibrium with BSA. No data appear to be available for the binding constants of elaidate and behenate to BSA. The data of Spector, John \& Fletcher (I969) suggest that fatty-acid chain length has a greater effect on the binding constants for both primary and secondary BSA binding sites than cis-unsaturation. Palmitate and palmitoleate were bound more tightly than myristate, oleate or stearate, and considerably more tightly than laurate. Thus, unless a trans double bond has a large effect on binding, it may be expected that elaidate would be bound considerably more tightly than either laurate or behenate.

The order and extent in which behenate enters the different lipid classes in strain y grown with elaidate and behenate is considered elsewhere (Plackett \& Rodwell, 1970). The results suggest that behenate is incorporated firstly into those lipids which are more rapidly turning over, i.e. lipid intermediates, and thence into the major lipid classes (glycolipids, phosphatidylglycerol and diphosphatidylglycerol), and that it is not incorporated extensively by a process of deacylation and reacylation of lipids already synthesized. The results suggest that behenate is incorporated firstly in the I-position of the glycerides; when all of these are occupied it is then incorporated into the 2-position (A. W. Rodwell, unpublished). McElhaney \& Tourtellotte (1970) investigated the positional distribution of esterified fatty acids in phosphatidylglycerol from Mycoplasma laidlawii. While some fatty acids had a marked positional preference, they could be incorporated into either position if supplied in excess.

The effects of a wide range of fatty acids on growth in a medium having a lower level of fatty-acid contamination, and possible explanations for the pronounced lag period in growth with certain pairs of fatty acids such as elaidate and stearate are considered in a paper now in preparation.

I am grateful to Mr B. Zwolak for skilled and diligent technical assistance.

\section{REFERENCES}

CotTew, G. S. (1970). Mycoplasmas isolated from cattle in Australia. Australian Veterinary Journal 46, 378-38r.

KopaCzyK, K. C. \& RadiN, N. S. (1965). In vivo conversions of cerebroside and ceramide in rat brain. Journal of Lipid Research 6, 140-145.

McElhaney, R. N. \& Tourtellotte, M. E. (I970). The relationship between fatty acid structure and the positional distribution of esterified fatty acids in phosphatidyl glycerol from Mycoplasma laidlawii $\mathbf{B}$. Biochimica et biophysica acta 202, $120-128$.

MoRris, L. J. (1964). Specific separations by chromatography on impregnated adsorbents. In New Biochemical Separations, pp. 295-319. Edited by A. T. James \& L. J. Morris. London: Van Nostrand.

PlacketT, P. \& Rodwell, A. W. (1970). Glycerolipid biosynthesis by Mycoplasma strain Y. Biochimica et biophysica acta 210, 230-240.

Rodwell, A.W. (1967). The nutrition and metabolism of Mycoplasma: progress and problems. Annals of the New York Academy of Sciences 143, 88-109.

RoDWeLl, A. W. (I968). Fatty-acid composition of Mycoplasma lipids: biomembrane with only one fatty acid. Science, New York r6o, 1350-1351.

Rodwell, A. W. (1969). The supply of cholesterol and fatty acids for the growth of mycoplasmas. Journal of General Microbiology 58, 29-37.

Spector, A. A., John, K. \& Fletcher, J. E. (1969). Binding of long-chain fatty acids to bovine serum albumin. Journal of Lipid Research 10, 56-67.

Spector, A. A., Steinberg, D. \& TANAKa, A. (1965). Uptake of free fatty acids by Ehrlich ascites tumor cells. Journal of Biological Chemistry 240, 1032-104I.

Turner, A. W., CAMpbell, A. D. \& Dick, A. T. (1935). Recent work on pleuropneumonia Contagiosa boum in North Queensland. Australian Veterinary Journal Ir, 63-7I. 